ORIGINAL ARTICLE

\title{
Effect of Dapsone Gel 7.5\% Compared to Tazarotene Gel 0.1\% for Treatment of Acne Vulgaris
}

\author{
Soad Mohamed Ibrahim ${ }^{1} *$; Zainab Tosson Labeeb $^{2}$; Amin Mohamed Amer ${ }^{2}$ \\ ${ }^{I}$ Dermatology, Venereology and Andrology Department, General Zagazig Hospital, Zagazig, Egypt \\ ${ }^{2}$ Dermatology, Venereology and Andrology Department, Faculty of Medicine, Zagazig University, \\ Zagazig, Egypt
}

\begin{abstract}
*Corresponding author : Soad Mohamed Ibrahim Dermatology, Venereology and Andrology Department, General Zagazig Hospital, E.mail: Zagazig, Egypt

soadn1212@gmail.com
\end{abstract}

$\begin{array}{ll}\text { Submit Date } & 2019-02-14 \\ \text { Revise Date } & 2019-05-19 \\ \text { Accept Date } & 2019-05-22\end{array}$

\begin{abstract}
Background: Dapsone $7.5 \%$ gel was well tolerated, with a low incidence of treatment-related adverse events, with the majority of adverse events being related to topical application and mild or moderate in severity.Tazarotene helps to normalize the abnormal keratinization in the follicular infundibulum and changes the microenvironment of the follicle to reduce the proliferation of $\mathrm{P}$. acnes. The aim of the study was to compare the efficacy and safety of dapsone gel $7.5 \%$ in relation to tazarotene gel $0.1 \%$ in the treatment of acne vulgaris. Methods: This study included 38 patients with different grades of $\mathrm{AV}$, of different sexes and their ages $\geq 12$ years, the patients were randomly selected from the outpatients clinics. Provided that the patients had not received any topical or systemic treatment for AV during the previous 2 weeks. A base line evaluation of the severity of acne was done by GAAS and lesional photography for each patient before the start of treatment with topical dapsone and tazarotene,then follow up at 2,4,8,12 weeks of treatment. Results: There is statistically significant difference between dapsone and tazarotene in side effects after treatment as $71.1 \%$ of the studied patients didn't complain of any side effects after dapsone while only $18.4 \%$ of the studied patients had no side effects after tazarotene. The response to treatment with dapsone on the right side and tazarotene on the left side among the studied acne patients showed mild improvement occured in $28.9 \%$ after dapsone and occured in $15.8 \%$ after tazarotene treatment, with no significant difference between both drugs. Conclusions: Dapsone $7.5 \%$ gel is a successful, effective and safe alternative in the treatment of acne vulgaris with insignificant side effects.
\end{abstract}

Keywords: Acne vulgaris; Tazarotene; Dapsone; Pathogenesis

\section{INTRODUCTION}

A cne vulgaris is chronic inflammatory disorder of pilosebaceous unit mainly characterized by comedones, papules and nodulocystic lesions affecting face and upper trunk [1]. The prevalence of acne vulgaris reported from various countries ranges to $87 \%$ of adolescents and up to $54 \%$ of adults. Though acne is self limiting disease, the presence of active acne lesions impacts the physical appearance and creates negative effects on psycho-social function for individual [2].

Acne vulgaris pathogenesis is characterized by excess sebum production, colonization of the follicular infundibula with Propionibacterium acnes, hyperkeratinization and production of inflammatory mediators. There is a complex interrelationship between P. acnes, sebaceous 
lipogenesis and inflammation [3]. P. acnes, a gram positive anaerobe that resides in the follicular infundibulum, enhance sebaceous lipogenesis. Squalene, a major component of sebum, can be oxidized in the presence of Ultra Violet light to convert into peroxidated squalene that has been demonstrated to induce production of inflammatory mediators in cultured keratinocytes. Then follicular occlusion and enhanced sebum retention leads to microcomedone formation [4].

Oral dapsone is effective in the treatment of severe, nodulocystic inflamed acne but haematological and other complications limits its use on routinely healthy acne patients. Topical dapsone is an alternative and showed good safety profile even in Glucose 6Phosphate Deficiency patients [5]. In vitro, a wide range of anti-inflammatory effects have been associated with dapsone including inhibition of: Inter Leukin 8 release from cultured keratinocytes; leukocyte migration ; calcium-dependent neutrophil function; release of prostaglandins, leukotrienes, and lysosomal acid hyrolases; and formation of 5lipoxygenase metabolites. Antibacterial activities are similar to sulfones [6].

Dapsone $7.5 \%$ gel is indicated for the oncedaily topical treatment of acne vulgaris in patients aged more than 12 years with moderate acne vulgaris, once-daily dapsone $7.5 \%$ gel reduced acne severity (as per the Global Acne Assessment Score) and lesion counts. The benefits of dapsone $7.5 \%$ gel were seen as early as week 2 for inflammatory lesion counts, and from week 4 or 8 for other outcomes [7]. Dapsone $7.5 \%$ gel was well tolerated, with a low incidence of treatment-related adverse events, with the majority of adverse events being administration-site related and mild or moderate in severity [8]. Tazarotene is a synthetic retinoid that has been approved by the United States Food and Drug Administration (FDA) for the treatment of acne vulgaris. Tazarotene is a prodrug that is converted to its active form, tazarotenic acid in the skin.
Tazarotene helps to normalize the abnormal keratinization in the follicular infundibulum and changes the microenvironment of the follicle to reduce the proliferation of $\mathrm{P}$. acnes [9].

\section{METHODS}

This study was carried out at the Dermatology, Venereology and Andrology Depratment, Zagazig University Hospitals during the period from march 2017 to october 2018.It included 38 patients with different grades of acne vulgaris, of different sexes and their ages $\geq 12$ years, the patients were randomly selected from the outpatients clinics. Provided that the patients had not received any topical or systemic treatment for AV during the previous 2 weeks. Written informed consents were taken from the patients. The study had the approval of the Institution Review Board (IRB) at Zagazig University.

This study included patients presenting with; Inflammatory and non-inflammatory acne vulgaris, both males and females are involved and the age of the patients more than 12 years.

Exclusion criteria included patients having Pregnancy, breast feeding or planning a pregnancy, history of hypersensitivity or allergy to dapsone, tazarotene gel or any of the study medication ingredients, the use of isotretinoin within 3 months before the beginning of the study (Figure 1). The work has been carried out in accordance with The Code of Ethics of the World Medical Association (Declaration of Helsinki) for studies involving humans.

Patients were subjected to the following: Personal history including age, sex, and previous treatment, history of AV including onset ,course and duration, history of systemic disease, general examination, complete dermatological examination including type of acne (mild, moderate and severe), onset course and duration of acne. Base line evaluation of the severity of acne was done by GAAS and lesional photography for each patient before the start of treatment with topical dapsone and tazarotene. 
Therapy of dapsone: Dapsone $7.5 \%$ aqueous gel Prepared by Department of Pharmaceutics, Faculty of Pharmacy, Zagazig University.

Preparation of dapsone gel 7.5\%: Every gram of dapsone gel $7.5 \%$ contains $75 \mathrm{mg}$ of dapsone in a fluid gel base of Carbomer 980, Diethylene glycol monoethylether (DGME). Methylparaben Sodium hydroxide and Purified water was added.

Each patient served as a control for himself. Each patient was instructed to use topical $7.5 \%$ dapsone gel once daily at night for 12 weeks duration on right hemi-face. Left hemi-face of each patient was treated with topical tazarotene gel once daily at night for 12 weeks duration. The patients were re-assessed at 2,4,6,8 and 12 weeks of therapy(the end of treatment) ,using GAAS and lesional photography .

Global Acne Assessment Score GAAS include:Clear (0): normal clear skin.Almost clear(1): rare non inflammatory lesions.Mild(2): some non inflammatory lesions with few inflammatory lesions (papules,pustules). Moderate (3): many comedones,papules,pustules. $\quad$ Severe(4): inflammatory lesions more apparent,many papules ,pustules and may be few nodulocystic lesions. Very severe (5): highly inflammatory lesions and many nodulocystic lesions ${ }^{[10]}$.

Patient's response to treatment was recorded as, good, moderate and mild.Good response if: there was clearence of the comedones, papules and pustules $>75 \%$. Moderate if: comedones, papules and pustules regressed in number or size by $50-75 \%$.Mild if: there was $25-50 \%$ decrease in comedones, papules and pustules number or size [11].

\section{Statistical analysis}

The collected data were analyzed by computer using Statistical Package of Social Services version 24 (SPSS). Data were represented in tables and graphs. Continuous quantitative variables e.g. age were expressed as the mean \pm SD \& median (range), and categorical qualitative variables were expressed as absolute frequencies (number) \& relative frequencies (percentage). Suitable statistical tests of significance were used after checked for normality. The results were considered statistically significant when the significant probability was less than $0.05(\mathrm{P}<0.05)$. P-value $<0.001$ was considered highly statistically significant (HS), and $\mathrm{P}$-value $\geq 0.05$ was considered statistically insignificant (NS).

\section{RESULTS}

The study included 38 patients ( 7 males and 31 females)as shwed in table 1 .Their ages ranged from 15 to 29 years old, with a mean of $20.34 \pm$ 3.58 years.Table 2 showed that there is statistically significant difference between dapsone and tazarotene in side effects after treatment as $71.1 \%$ of the studied patients didn't complain of any side effects after dapsone while only $18.4 \%$ of the studied patients had no side effects after tazarotene.Table 3showed the response to treatment with Dapsone on the rt side and Tazarotene on the lt side among the studied acne patients where mild improvement occured in $28.9 \%$ after Dapsone and occured in $15.8 \%$ after Tazarotene treatment, with no significant difference between both drugs. Table 4,5 showed that there is statistically significant change in Global Acne assessment score after treatment with Dapsone where clear and almost clear score is higher after treatment than before treatment $(23.7 \%$ vs zero\%) \& $(44.7 \%$ vs zero\%) respectively. Table S1 showed that there is statistically significant change in Global Acne assessment score after treatment with Tazarotene where clear and almost clear score is higher after treatment than before treatment $(26.3 \%$ vs zero\%) \& $(52.6 \%$ vs zero $\%)$ respectively.Severe cases used combination of topical and systemic treatment. Table S2 showed Global Acne Assessment Score after treatment with dapsone on the $\mathrm{rt}$ side and tazarotene on the lt side among the studied acne patients showed clear score in $23.7 \%$ after dapsone and occurs in $26.3 \%$ after tazarotene treatment, with no significant difference between both drugs. 
Table 1.Demographic data of the acne patients $(\mathrm{N}=38)$.

\begin{tabular}{|l|c|c|}
\hline Demographic data & \multicolumn{2}{|c|}{ Studied patients $(\mathbf{N}=38)$} \\
\hline Age (years) & No. & \% \\
\hline Mean \pm SD & \multicolumn{2}{|c|}{$20.34 \pm 3.58$} \\
\hline Median (Range) & \multicolumn{2}{|c|}{$19.5(15-29)$} \\
\hline Sex & 7 & 18.4 \\
\hline Male & 31 & 81.6 \\
\hline Female
\end{tabular}

Table 2.Side effects after treatment with Dapsone on the right side and Tazarotene on the left side among the studied acne patients $(\mathrm{N}=38)$.

\begin{tabular}{|c|c|c|c|c|c|}
\hline \multirow[t]{2}{*}{ Side effects } & \multicolumn{2}{|c|}{$\begin{array}{l}\text { on right side } \\
\text { Dapsone }\end{array}$} & \multicolumn{2}{|c|}{$\begin{array}{l}\text { on left side } \\
\text { Tazarotene }\end{array}$} & \multirow[t]{2}{*}{$\begin{array}{l}\text { p- value } \\
\text { of fisher's }\end{array}$} \\
\hline & No. & $\%$ & No. & $\%$ & \\
\hline No side effects & 27 & 71.1 & 7 & 18.4 & $0.000 *$ \\
\hline Irritation & 11 & 28.9 & 20 & 52.6 & $0.035 *$ \\
\hline Erythema & 0 & 0.0 & 14 & 36.8 & $0.000 *$ \\
\hline Burning sensation & 0 & 0.0 & 9 & 23.7 & $0.002 *$ \\
\hline Desquamation & 0 & 0.0 & 3 & 7.9 & 0.240 \\
\hline
\end{tabular}

Table 3.Response to treatment with Dapsone on the rt side and Tazarotene on the lt side among the studied acne patients $(\mathrm{N}=38)$.

\begin{tabular}{|l|c|c|c|c|c|c|}
\hline \multirow{2}{*}{\begin{tabular}{l} 
Improvement \\
\cline { 2 - 7 }
\end{tabular}} & \multicolumn{2}{|c|}{$\begin{array}{c}\text { on right side } \\
\text { Dapsone }\end{array}$} & \multicolumn{2}{c|}{$\begin{array}{c}\text { on left side } \\
\text { Tazarotene }\end{array}$} & $\begin{array}{c}\text { chi - } \\
\text { square } \\
\text { test }\end{array}$ & p- value \\
\hline Mild improvement & 11 & 28.9 & 6 & 15.8 & & \\
\hline $\begin{array}{l}\text { Moderate } \\
\text { improvement }\end{array}$ & 13 & 34.2 & 16 & 42.1 & 1.914 & 0.384 \\
\hline Good improvement & 14 & 36.8 & 16 & 42.1 & & (NS) \\
\hline
\end{tabular}

Table 4. Global Acne assessment score before treatment among the studied acne patients $(\mathrm{N}=38)$.

\begin{tabular}{|l|c|c|}
\hline GAAS & \multicolumn{2}{|c|}{$\begin{array}{c}\text { studied acne patients } \\
\text { (N=38) }\end{array}$} \\
\hline Mild & No. & $\mathbf{\%}$ \\
\hline Moderate & 12 & 31.6 \\
\hline Severe & 24 & 63.2 \\
\hline
\end{tabular}


Table 5.Global Acne assessment score after treatment with Dapsone on the rt side among the studied acne patients $(\mathrm{N}=38)$.

\begin{tabular}{|c|c|c|c|c|c|}
\hline \multirow[t]{2}{*}{ GAAS } & \multicolumn{2}{|c|}{ Before Dapsone } & \multicolumn{2}{|c|}{ After Dapsone } & \multirow{2}{*}{$\begin{array}{l}\text { p-value of } \\
\text { Freidman } \\
\text { test }\end{array}$} \\
\hline & No. & $\%$ & No. & $\%$ & \\
\hline Clear & 0 & 0.0 & 9 & 23.7 & \multirow{5}{*}{$\begin{array}{c}\text { 0.000* } \\
\text { (HS) }\end{array}$} \\
\hline Almost clear & 0 & 0.0 & 17 & 44.7 & \\
\hline Mild & 12 & 31.6 & 12 & 31.6 & \\
\hline Moderate & 24 & 63.2 & 0 & 0.0 & \\
\hline Severe & 2 & 5.3 & 0 & 0.0 & \\
\hline
\end{tabular}

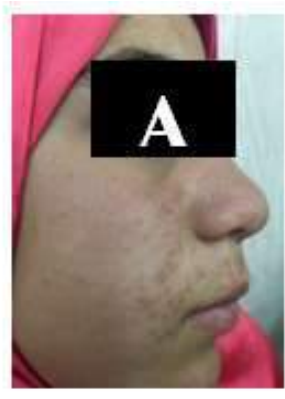

Figure 1. (A) Female patient (right side) before treatmen

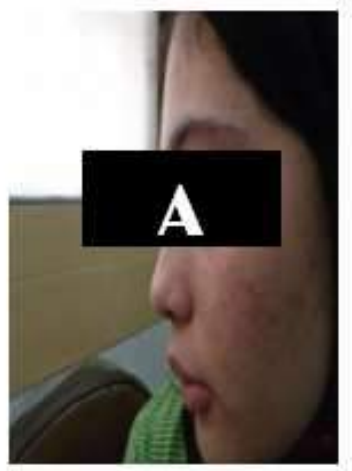

Figure 2. (A) Female patient (left side) with moderate acne vulgaris before treatment by tazarotenegel.

\section{DISCUSSION}

Acne vulgaris (AV) is the most common cutaneous disorder seen in dermatology practice among adult populations [12]. Data from two identically designed pivotal studies of a new, once-daily formulation of topical

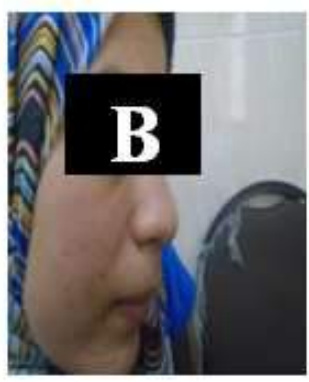

(B) The same patient at the end of. treatment by dapsonegel showing good response.

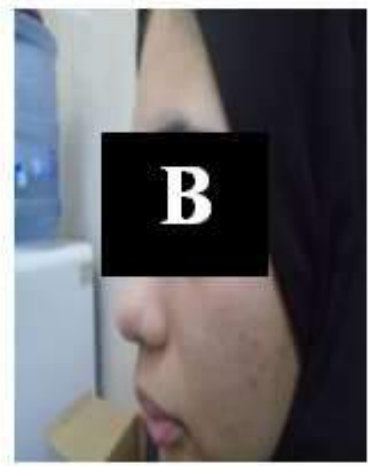

(B) The same patient at the end of treatment showing good response.

dapsone gel, $7.5 \%$ demonstrated safety and efficacy versus vehicle $[13,14]$. The new formulation of dapsone gel, $7.5 \%$ recently gained approval from the United States Food and Drug Administration for the once-daily treatment of acne vulgaris [15]. Tazarotene is 
approved for the treatment of psoriasis and acne. Only $0.1 \%$ strength is approved by the United States Food and Drug Administration (FDA) for the treatment of acne. Tazarotene cream $0.1 \%$ is indicated for acne vulgaris, and gel $0.1 \%$ is indicated for mild-to-moderate acne vulgaris, whereas foam $0.1 \%$ is indicated for moderate-to-severe acne. Comparison studies between tazarotene, adapalene, and tretinoin report mixed results, with some researchers suggesting comparable efficacy and tolerability, while others report greater efficacy and irritation with tazarotene [16].

Tazarotene is a synthetic retinoid that has been approved by the United States Food and Drug Adminstration FDA for the treatment of acne vulgaris. Tazarotene helps to normalize the abnormal keratinization in the follicular infundibulum and changes the microenvironment of the follicle to reduce the proliferation of P. acnes [17]. This study was established to compare the efficacy and safety of topical dapsone $7.5 \%$ gel for the treatment of AV on 38 patients of different sexes, ages and with different grades of acne. They were 7 males(18\%) and 31 females $(82 \%)$, patients act as control for themselves by applying dapsone gel $7.5 \%$ on their right side of the face and tazarotene gel $0.1 \%$ on the left side of the face. Duration of acne among the studied patients ranged from 1 month to 3 years, with a mean \pm SD of age was $20.34 \pm 3.58$ years, the mean \pm $\mathrm{SD}$ of the duration of acne was $12.26 \pm 6.12$, and $57.9 \%$ of the studied patients suffered from acne for less than 9 months, while $42.1 \%$ of them have acne for 9 months and more.

Before treatment, $63.2 \%$ of the studied patients have moderate acne according to Global Acne assessment score while only $5.3 \%$ are severe. After treatment, there is statistically significant change in Global Acne Assessment Score after treatment with Dapsone where clear and almost clear score is higher after treatment than before treatment $(23.7 \%$ vs zero\%) \& $(44.7 \%$ vs zero\%) respectively. Also there is statistically significant change in Global Acne assessment score after treatment with Tazarotene where clear and almost clear score is higher after treatment than before treatment $(26.3 \%$ vs zero\%) \& (52.6\% vs zero\%) respectively. Global Acne Assessment Score after treatment with Dapsone on the rightt side and Tazarotene on the left side among the studied acne patients where clear score in $23.7 \%$ after Dapsone and occurs in $26.3 \%$ after Tazarotene treatment, with no significant difference between both drugs. There is statistically significant difference between dapsone and tazarotene in side effects after treatment as $71.1 \%$ of the studied patients didn't complain of any side effects after dapsone while only $18.4 \%$ of the studied patients have no side effects after tazarotene, erythema and burning sensation are more statistically higher after tazarotene treatment than dapsone ( $26.3 \%$ vs zero $\%)$ and (23.7\% Vs zero \%) respectively.

Response to treatment with dapsone on the right side and tazarotene on the left side among the studied acne patients where mild improvement occurs in $28.9 \%$ after dapsone and occurs in $15.8 \%$ after tazarotene treatment, with no significant difference between both drugs. In agreement with this study, El-Tonsy et al. [18] studied the mean duration of acne in the study population was 29.27 months. In relation to response to treatment we found that the study shows that mild improvement, moderate improvement and good improvement occurs in $28.9 \%, 34.2 \%$ \& $36.8 \%$ respectively after dapsone treatment, while after Tazarotene mild improvement, moderate improvement and good improvement occurs in $15.8 \%, 42.1 \%$ \& $42.1 \%$ respectively. According to Del Rosso et al., [19] There were fifteen reports of erythema, dryness, oiliness, pruritus, or burning effects after Dapsone.

Similarly, adverse events were reported for 18.3 percent $(396 / 2161)$ of patients who received dapsone gel, $7.5 \%$ and 18.8 percent (409/2175) of patients who received vehicle. Most adverse events were mild to moderate in severity. These events consisted of application site dryness, 
application site pruritus, and application site pain. The incidence of these events for patients who received dapsone gel $7.5 \%$ was similar to that for patients who received vehicle according to Thiboutot et al [20]. But according to Feldman et al. [21], Most adverse reactions after Tazarotene were mild to moderate in severity. Severe adverse reactions occurred in $3.0 \%$ of subjects; $2.6 \%$ of patients discontinued due to local skin reactions. There is no significant difference between response after dapsone treatment and age or sex of the studied patients .As regard side effects there is statistically significant difference between dapsone and tazarotene in side effects after treatment as $71.1 \%$ of the studied patients didn't complain of any side effects after dapsone while only $18.4 \%$ of the studied patients have no side effects after tazarotene, irritation, erythema and burning sensation are more statistically higher after tazarotene gel treatment than dapsone $(52.6 \%$ vs $28.9 \%)$ $\&(36.8 \%$ vs zero\%) and $(23.7 \%$ Vs zero \%) respectively.

\section{CONCLUSION}

According to our results, we suggest that dapsone $7.5 \%$ gel can be a successful, effective and safe alternative in the treatment of acne vulgaris with insignificant side effects.

We recommend trying dapsone $7.5 \%$ gel on a greater number of patients for longer duration of treatment and to follow up cases after improvement to assess the recurrence of lesions after stoppage of the treatment. Further studies are required for standardization of dapsone gel use in acne and the possibility of combining it with other medications for achieving better results.

Declaration of interest

The authors report no conflicts of interest. The authors alone are responsible for the content and writing of the paper.

Funding information

None declared

Tables S1, 2 are shown on the online supplement

\section{REFERENCES}

1. Demircay Z, Seckin D, Senol A, Demir F(2016). Patient's perspective: an important issue not to be overlooked in assessing acne severity. Eur $\mathrm{J}$ Dermatol. 18(2):181-4.

2. Goulden V, Stables GI, Cunliffe WJ (2011) Prevalence of facial acne in adults. J Am Acad Dermatol. 41:577-80.

3. BELLEW, Susun; THIBOUTOT, Diane; DEL, JQ Rosso (2011) Pathogenesis of acne vulgaris: what's new, what's interesting and what may be clinically relevant. J Drugs Dermatol. 10(6):5825.

4. Ottaviani, M, Alestas T, Flori E. Mastrofrancesco A, Zouboulis CC, Picardo M. Peroxidated squalene induces the production of inflammatory mediators in $\mathrm{HaCaT}$ keratinocytes: a possible role in acne vulgaris? J Invest Dermatol 2012; 126:2430-7.

5. Stotland M, Shalita AR, Kissling RF. Dapsone 7.5\% gel: a review of its efficacy and safety in the treatment of acne vulgaris. Am J Clin Dermatol $2014 ; 10: 221-7$.

6. Jeremy AH, Holland DB, Roberts SG, Thomson KF, Cunliffe WJ. Inflammatory events are involved in acne lesion Initiation. $\mathrm{J}$ Invest Dermatol 2013; 121: 20-7.

7. Raimer S, Maloney JM, Bourcier M, Wilson D, Papp K, Siegfried E, et al. Efficacy and safety of dapsone gel $5 \%$ for the treatment of acne vulgaris in adolescents. Cutis 2016; 81: 171-8.

8. Pickert A, Raimer S. An evaluation of dapsone gel 5\% in the treatment of acne vulgaris. Expert Opin Pharmacother 2016; 10: 1515-21.

9. Talpur R, Cox K, Duvic M. Efficacy and safety of topical tazarotene: a review. Expert Opin Drug Metab Toxicol 2017; 5(2): 195-210.

10. Becker M, Wild T, Zouboulis CC.: Objective assessment of acne. Clinics in dermatology 2017; 35: 147-155.

11. Rao PK, Bhat RM., Nandakishore B, Dandakeri S, Martis J, Kamath GH. Safety and efficacy of lowdose isotretinoin in the treatment of moderate to severe acne vulgaris. Ind $\mathrm{j}$ dermatol 2014; 59.3: 316.

12. Davis SA, Narahari S, Feldman SR, Huang W, Pichardo-Geisinger RO, McMichael AJ. Top dermatologic conditions in patients of color: an analysis of nationally representative data. J Drugs in Dermatol 2012; 11: 466-473.

13. Stein LG, Jarratt MT, Bucko AD, Grekin SK, Berlin M, Bukhalo M, et al. Efficacy and Safety of 
Once-Daily Dapsone Gel, 7.5\% for Treatment of Adolescents and Adults With Acne Vulgaris: First of Two Identically Designed, Large, Multicenter, Randomized, Vehicle-controlled Trials. J Drugs in Dermatol 2016; 15: 553-561.

14. Eichenfield LF, Lain T, Frankel EH, Jones TM, Chang-Lin JE, Berk DR, et al. Efficacy and Safety of Once-Daily Dapsone Gel, 7.5\% for Treatment of Adolescents and Adults With Acne Vulgaris: Second of Two Identically Designed, Large, Multicenter, Randomized, Vehicle-Controlled Trials. J Drugs in dermatol 2016; 15: 962-969.

15. Gel A. 7.5\% [package insert]. Dublin, Ireland: Allergan plc, 2016.

16. Pariser D, Colon LE, Johnson LA, Gottschalk RW. Adapalene $0.1 \%$ gel compared to tazarotene $0.1 \%$ cream in the treatment of acne vulgaris. J Drugs in Dermatol 2008; S18-23.

17. Talpur R, Cox K, Duvic M. Efficacy and safety of topical tazarotene: a review. Expert Opin Drug Metab Toxicol 2017; 5(2): 195-210.
18.El-Tonsy TMK, Mohammed MA, Hamed EE, Abou Y, Tawfik SH. Bacteriological study of Acne Vulgaris in Cairo Egypt. Egy J HosMed $2018 ; 72$.

19. Del Rosso JQ, Kircik L, Tanghetti E. Management of Truncal Acne Vulgaris with Topical Dapsone 7.5\% Gel. J Clinical Aesthetic Dermatol 2018; 11: 45.

20. Thiboutot DM, Kircik L, McMichael A, CookBolden FE, Tyring SK., Berk DR, et al. Efficacy, safety, and dermal tolerability of dapsone gel, 7.5\% in patients with moderate acne vulgaris: a pooled analysis of two phase 3 trials. J Clin Aesthetic Dermato 2016; 9: 18.

21. Feldman SR, Werner CP, Alio AS. The efficacy and tolerability of tazarotene foam, $0.1 \%$, in the treatment of acne vulgaris in 2 multicenter, randomized, vehicle-controlled, double-blind studies. J Drugs Dermatol 2013; 12: 438-446.

To Cite This Article: Ibrahim SM, Labeeb ZT, Amer AM. Effect of Dapsone Gel 7.5\% Compared to Tazarotene Gel $0.1 \%$ for Treatment of Acne Vulgaris. ZUMJ 2019; 25(5): 657-664. Doi: 10.21608/zumj.2019.7618.10400. 